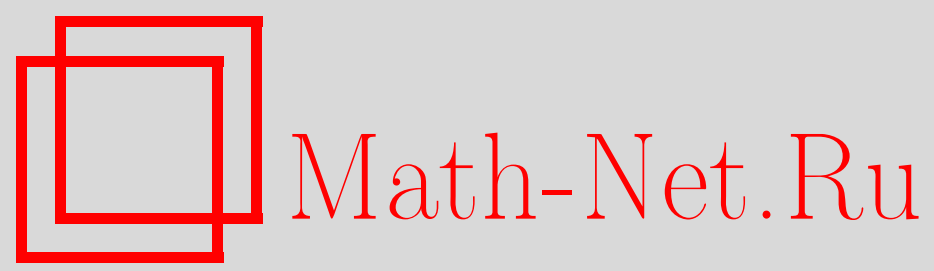

И. Я. Арефьева, И. В. Волович, О нелокальных космологических уравнениях на полуоси, Вестн. Сам. гос. техн. ун-та. Сер. Физ.мат. науки, 2011, выпуск 1(), 16-27

DOI: https://doi.org/10.14498/vsgtu938

Использование Общероссийского математического портала Math-Net.Ru подразумевает, что вы прочитали и согласны с пользовательским соглашением http://www.mathnet.ru/rus/agreement

Параметры загрузки:

IP : 3.80 .181 .102

26 апреля 2023 г., 14:59:06 
УДК 517.956.4:524.834

\title{
О НЕЛОКАЛЬНЫХ КОСМОЛОГИЧЕСКИХ УРАВНЕНИЯХ НА ПОЛУОСИ
}

\author{
И. Я. Арефъева, И. В. Волович
}

Математический институт им. В. А. Стеклова РАН, 119991, Москва, ул. Губкина, 8.

E-mails: arefeva@mi.ras.ru, volovich@mi.ras.ru

\begin{abstract}
Рассмотрена система нелокалъных космологических уравнений на полуоси по времени, которые более адекватно описъвают динамику развития Вселенной, чем обсуждавшиеся ранее уравнения на всей оси, поскольку метрика Фридмана сингулярна в начальный момент времени. Установлено, что в определение нелокального экспоненииального оператора, входящего в рассматриваемые уравнения, входит дополнительная произвольная функиия, отсутствовавшая в уравнениях на всей оси. Показано, что эта функиия может быть выбрана таким образом, чтобы один из параметров хаотической инфляиии становился сколь угодно малым. Построены решения линеаризованных нелокальных уравнений на nолуоси.
\end{abstract}

Ключевые слова: уравнения с бесконечным числом производных, космологические модели, уравнение теплопроводности.

Введение. Уравнения струнной теории поля с бесконечным числом производных, т. е. нелокальные интегральные уравнения определенного вида, было предложено использовать в космологии в работе [1]. В дальнейшем уравнения такого типа исследовались в многочисленных работах (см. [2-18], а также ссылки в работах $[19,20])$. Простейший оператор с бесконечным числом производных имеет вид экспоненциального оператора $\exp \square$, где $\square-$ волновой оператор. Нелинейные уравнения с таким оператором возникают в $p$-адической теории поля [21-25], для случая плоской метрики и зависимости от одной переменной они исследовались в [26-30].

В математической литературе линейные уравнения с бесконечным числом производных рассматривались в [31-34]. Нелинейные интегральные уравнения на полуоси изучались в $[35,36]$, где приводятся также ссылки на другие работы.

Обычно предполагалось, что переменная времени в нелокальных космологических уравнениях пробегала всю вещественную ось. Заметим однако, что в решениях космологических уравнений Фридмана метрика содержит сингулярность в начальный момент времени (см., например, [37]), поэтому представляется более естественным рассматривать уравнения на полуоси по времени. В настоящей работе рассмотрена система нелокальных космологических уравнений на полуоси по времени, которые более адекватно описывают динамику развития Вселенной, чем обсуждавшиеся ранее уравнения на всей оси. Установлено, что для уравнений на полуоси возникает новое интересное явление, отсутствующее у уравнений на всей оси - само определение экспоненциального оператора, входящего в рассматриваемые уравнения, содержит дополнительную произвольную функцию (источник $J$ ), отсутство-

Ирина Ярославна Арефъева (д.ф.-м.н.), профессор, ведущий научный сотрудник, отд. теоретической физики. Игорь Васильевич Волович (д.ф.-м.н., член-корр. РАН), заведующий отделом, отд. математической физики. 
вавшую в уравнениях на всей оси. Показано, что эта функция может быть выбрана таким образом, чтобы один из параметров в моделях хаотической инфляции становился сколь угодно малым. Этот результат может оказаться полезным для расширения допустимой области изменения параметров потенциалов, изучаемых в моделях хаотической инфляции. Построены решения линеаризованных нелокальных уравнений.

Нелокальные уравнения на полуоси рассматривались в [13] при помощи метода преобразования Лапласа, однако в этом методе дополнительная произвольная функция не возникает.

Действие, предложенное в [1], имеет вид

$$
S=\int d^{4} x \sqrt{-g}\left\{M_{p}^{2} \frac{R}{2}+\frac{1}{\alpha^{\prime 2} g_{0}^{2}}\left(\frac{\alpha^{\prime}}{2} \Phi\left(\square+\frac{1}{\alpha^{\prime}}\right) e^{-\kappa \alpha^{\prime} \square} \Phi-V(\Phi)\right)\right\} .
$$

Здесь $\square=\frac{1}{\sqrt{-g}} \partial_{\mu} \sqrt{-g} g^{\mu \nu} \partial_{\nu}-$ волновой оператор, $g_{\mu \nu}$ - метрический тензор, $M_{p}$ - масса Планка, $\Phi=\Phi(x)$ - скалярное поле, $\alpha^{\prime}-$ квадрат характерной длины струны, $V(\Phi)$ - потенциал взаимодействия, $V(\Phi)=\Phi^{3} / 3$ для бозонной струны, $V(\Phi)=\Phi^{4} / 4$ для фермионной струны, $g_{0}^{2}$ - безразмерная константа связи, $\kappa=2 \ln (4 / 3 \sqrt{3})$ - численный параметр.

Действие для $p$-адической струны в гравитационном поле имеет вид [1]

$$
S=\int d^{4} x \sqrt{-g}\left\{M_{p}^{2} \frac{R}{2}+\frac{1}{\alpha^{\prime 2} g_{p}}\left(\Phi e^{-\kappa_{p} \alpha^{\prime} \square} \Phi-V(\Phi)\right)\right\} .
$$

Здесь $g_{p}$ и $\kappa_{p}$ - постоянные, $V(\Phi)=\Phi^{p+1} /(p+1)$.

Простейшее локальное действие с произвольным источником имеет вид

$$
S=\int d^{4} x \sqrt{-g}\left\{\frac{M_{P}^{2}}{2} R+\frac{1}{2} \phi \square \phi-U(\phi)+J \phi\right\},
$$

где $J=J(x)$ - произвольный источник и $U(\phi)$ - потенциал взаимодействия. Действия такого типа естественно возникают как результат анализа нелокальных операторов на полуоси, выполненного в данной работе.

В следующем разделе дано определение нелокального оператора на полуоси при помощи решения уравнения теплопроводности с произвольной функцией на границе. В разделе 2 приводятся решения линеаризованных уравнений. В разделе 3 сформулированы нелокальные уравнения на полуоси во Фридмановской космологии и построены решения линеаризованных уравнений. В разделе 4 показано, что для инфляционного космологического сценария при помощи выбора функции источника один из параметров медленного скатывания может быть сделан сколь угодно малым, что может представлять интерес при выборе космологических моделей.

1. Нелокальный оператор и уравнение теплопроводности. Для пространственно-однородных конфигураций в пространстве Минковского действие волнового оператора сводится к дифференцированию по времени: $\square=-\partial^{2} / \partial t^{2}$. Мы хотим придать математический смысл выражению $e^{\partial_{t}^{2}}$. Будем следовать методу Фока вспомогательного параметра [38-40], известного также как метод уравнения теплопроводности. Введём формально функцию двух переменных $\Psi(\tau, t)=e^{\tau \partial_{t}^{2}} \varphi(t)$. Эта функция удовлетворяет уравнению теплопроводности $[26,29,27,7]$

$$
\left(\partial_{\tau}-\partial_{t}^{2}\right) \Psi(t, \tau)=0,
$$


и начальному условию

$$
\left.\Psi(t, \tau)\right|_{\tau=0}=\varphi(t) .
$$

Предположим теперь, что дана функция $\Psi(\tau, t)$, удовлетворяющая уравнению (3) и граничному условию (4). Тогда действие оператора $e_{t}^{2}$ на функцию $\varphi(t)$ определяется следующим образом:

$$
\left.e^{\partial_{t}^{2}} \varphi(t) \equiv \Psi(t, \tau)\right|_{\tau=1}
$$

Имеется, как известно, важное различие в постановке краевых условий для уравнения теплопроводности на всей прямой и на полуоси. Если переменная $t$ пробегает всю вещественную ось, то для единственности решения уравнения (3) в соответствующих функциональных пространствах достаточно наложить условие (4). Однако при рассмотрении задачи на полуоси $0<t<\infty$ требуется ещё дополнительное условие при $t=0$ [41]. Таким образом, на полуоси появляется дополнительная произвольная функция, что приводит к важным последствиям для космологии, как будет показано ниже.

Рассмотрим следующую смешанную начально-краевую задачу Дирихле для уравнения теплопроводности на полуоси:

$$
\left\{\begin{array}{l}
\frac{\partial}{\partial \tau} \Psi_{D}(t, \tau)=\frac{\partial^{2}}{\partial t^{2}} \Psi_{D}(t, \tau), \quad t>0, \quad \tau>0 \\
\Psi_{D}(t, 0)=\varphi(t), \quad \Psi_{D}(0, \tau)=\mu(\tau) .
\end{array}\right.
$$

Функция $\mu(\tau)$ задаёт граничные условия (температуру на границе) при $t=0$. Индекс $D$ у функции $\Psi_{D}(t, \tau)$ означает «Дирихле».

Мы определим теперь действие оператора $e^{\tau \partial_{t}^{2}}$ на полуоси следующим образом:

$$
e^{\tau \partial_{t}^{2}} \varphi(t)=\Psi_{D}(t, \tau), \quad t>0, \quad \tau>0
$$

где $\Psi_{D}(t, \tau)$ решение задачи (5).

Как известно [41], решение задачи (5) имеет следующий вид:

$$
\begin{aligned}
\Psi_{D}(t, \tau)=\frac{1}{\sqrt{4 \pi \tau}} \int_{0}^{\infty} \varphi\left(t^{\prime}\right)\left[e^{-\frac{\left(t-t^{\prime}\right)^{2}}{4 \tau}}-e^{-\frac{\left(t+t^{\prime}\right)^{2}}{4 \tau}}\right] d t^{\prime}+ & \\
& +\frac{t}{\sqrt{4 \pi}} \int_{0}^{\tau} \frac{\mu\left(\tau^{\prime}\right)}{\left(\tau-\tau^{\prime}\right)^{3 / 2}} e^{-\frac{t^{2}}{4\left(\tau-\tau^{\prime}\right)}} d \tau^{\prime}
\end{aligned}
$$

Наше определение действия оператора $e_{D, J}^{\tau \partial_{t}^{2}}$ может быть записано в виде

$$
e_{D, J}^{\tau \partial_{t}^{2}} \varphi(t)=e_{D, 0}^{\tau \partial_{t}^{2}} \varphi(t)+J(t, \tau)
$$

где

$$
e_{D, 0}^{\tau \partial_{t}^{2}} \varphi=\frac{1}{2 \sqrt{\tau \pi}} \int_{0}^{\infty} d t^{\prime}\left[e^{-\frac{\left(t-t^{\prime}\right)^{2}}{4 \tau}}-e^{-\frac{\left(t+t^{\prime}\right)^{2}}{4 \tau}}\right] \varphi\left(t^{\prime}\right)
$$

и функция $J$ (источник) имеет вид

$$
J(t, \tau)=\frac{t}{\sqrt{4 \pi}} \int_{0}^{\tau} \frac{\mu\left(\tau^{\prime}\right)}{\left(\tau-\tau^{\prime}\right)^{3 / 2}} e^{-\frac{t^{2}}{4\left(\tau-\tau^{\prime}\right)}} d \tau^{\prime}
$$


Здесь индекс $J$ указывает на зависимость данного определения от выбора функции $J$.

Рассмотрим также задачу Неймана для уравнения теплопроводности на полуоси $t>0$ :

$$
\left\{\begin{array}{l}
\frac{\partial}{\partial \tau} \Psi_{N}(t, \tau)=\frac{\partial^{2}}{\partial t^{2}} \Psi_{N}(t, \tau), \quad t>0, \quad \tau>0 \\
\Psi_{N}(t, 0)=\varphi(t),\left.\quad \frac{\partial}{\partial t} \Psi_{N}(t, \tau)\right|_{t=0}=\nu(\tau)
\end{array}\right.
$$

Решение задачи (7) имеет вид

$$
\begin{aligned}
\Psi_{N}(t, \tau)=\frac{1}{\sqrt{4 \pi t}} \int_{0}^{\infty} \varphi\left(t^{\prime}\right)\left[e^{-\frac{\left(t-t^{\prime}\right)^{2}}{4 \tau}}+e^{-\frac{\left(t+t^{\prime}\right)^{2}}{4 \tau}}\right] d t^{\prime}- & \\
& -\frac{1}{\sqrt{\pi}} \int_{0}^{t} \frac{\nu\left(\tau^{\prime}\right)}{\left(\tau-\tau^{\prime}\right)^{1 / 2}} e^{-\frac{t^{2}}{4\left(\tau-\tau^{\prime}\right)}} d \tau^{\prime} .
\end{aligned}
$$

Мы определим оператор $e^{\tau \partial_{t}^{2}}$ на полуоси с граничными условиями Неймана следующим образом:

$$
e_{N, J}^{\tau \partial_{t}^{2}} \varphi(t)=\Psi_{N}(t, \tau)
$$

Здесь $N$ означает граничные условия Неймана, а функция источника $J$ есть

$$
J(t, \tau)=\frac{1}{\sqrt{\pi}} \int_{0}^{t} \frac{\nu\left(\tau^{\prime}\right)}{\left(\tau-\tau^{\prime}\right)^{1 / 2}} e^{-\frac{t^{2}}{4\left(\tau-\tau^{\prime}\right)}} d \tau^{\prime} .
$$

\section{2. Решение линеаризованных нелокальных уравнений на полуоси.}

2.1. Граничные условия Дирихле без источника. Рассмотрим сначала случай граничных условий Дирихле с исчезающим источником $J(t, \tau)=0$. В этом случае уравнение

$$
e_{D, 0}^{\tau \partial_{t}^{2}} \Phi(t)=m^{2} \Phi(t), \quad t>0,
$$

где $m>0$ и $\tau>0$ - некоторые параметры, имеет вид следующего интегрального уравнения:

$$
\frac{1}{2 \sqrt{\tau \pi}} \int_{0}^{\infty} d t^{\prime}\left[e^{-\frac{\left(t-t^{\prime}\right)^{2}}{4 \tau}}-e^{-\frac{\left(t+t^{\prime}\right)^{2}}{4 \tau}}\right] \Phi\left(t^{\prime}\right)=m^{2} \Phi(t), \quad t>0 .
$$

Уравнение (8) может быть решено, если выполнить нечётное продолжение функции $\Phi(t)$ на всю вещественную ось. Тогда получим уравнение

$$
e^{\tau \partial_{t}^{2}} \Phi(t)=m^{2} \Phi(t), \quad t \in \mathbb{R} .
$$

Нечётное решение уравнения (9) имеет вид

$$
\Phi_{D}(t)=\sum_{n=-\infty}^{\infty}\left(\frac{1}{2} B_{n}\left(e^{\alpha_{n} t}-e^{-\alpha_{n} t}\right)+\frac{1}{2} B_{n}^{*}\left(e^{\alpha_{n}^{*} t}-e^{-\alpha_{n}^{*} t}\right)\right),
$$


где $B_{n}$ - произвольные константы, а $\alpha_{n}$ - решения уравнения

$$
e^{\tau \alpha_{n}^{2}}=m^{2}, \quad \text { где } \quad \alpha_{n}= \pm \sqrt{\frac{\ln m^{2}+2 \pi i n}{\tau}}, \quad n \in \mathbb{Z} .
$$

Таким образом, общее решение уравнения (8) имеет вид (10).

Особый интерес представляет рассмотрение $\alpha_{0}$ моды. В этом случае

$$
\Phi(t)=B_{0} \operatorname{sh} \alpha_{0} t
$$

и мы имеем соотношение

$$
e_{D, 0}^{\tau \partial_{t}^{2}} \operatorname{sh} r t=e^{\tau r^{2}} \operatorname{sh} r t, \quad t>0 ; e^{\tau r^{2}}=m^{2}
$$

или

$$
\frac{1}{2 \sqrt{\tau \pi}} \int_{0}^{\infty} d t^{\prime}\left[e^{-\frac{\left(t-t^{\prime}\right)^{2}}{4 \tau}}-e^{-\frac{\left(t+t^{\prime}\right)^{2}}{4 \tau}}\right] \operatorname{sh} r t^{\prime}=e^{\tau r^{2}} \operatorname{sh} r t .
$$

Энергия и давление на этом решении имеют вид [5]

$$
E=-\frac{m^{2} \ln m^{2}}{2} B_{0}^{2}, \quad p=-\frac{m^{2} \ln m^{2}}{2} e^{2 \alpha_{0}^{2}} B_{0}^{2} \operatorname{ch}\left(2 \alpha_{0} t\right),
$$

где мы положили $\tau=2$ и $g_{4}=1$. Энергия является константой, но давление зависит от времени. Если $m^{2}>1$, то энергия $E$ отрицательная, но выражение $-(E+p)-$ положительно:

$$
-\frac{1}{2 m_{p}^{2}}(E+p)=\frac{m^{2}}{2 m_{p}^{2}} \frac{\ln m^{2}}{2} B_{0}^{2}\left(1+\operatorname{ch}\left(2 \alpha_{0} t\right)\right) .
$$

Заметим, что если сделать наивное локальное приближение к (1), то мы получим массивное гостовское поле, так что появление отрицательной энергии не является неожиданным. Если в (1) положить $g=-1$, то этот случай будет соответствовать обычному массивному полю и мы получим положительную энергию.

Можно показать, что для граничных условий Неймана знаки энергии будут противоположными.

2.2. Граничные условия Дирихле с источником. Рассмотрим теперь нелокальное линейное уравнение с граничными условиями Дирихле при наличии источника:

$$
e_{D, J}^{\tau \partial_{t}^{2}} \Phi=m^{2} \Phi .
$$

Это уравнение можно переписать в виде

$$
e_{D, 0}^{\tau \partial_{t}^{2}} \Phi=-J(\tau, t)+m^{2} \Phi .
$$

Построим решение последнего уравнения для некоторого специального источника $J$. 
Действие оператора $e_{D, 0}^{\tau \partial_{t}^{2}}$ на постоянную $\Phi_{0}$ имеет следующий вид:

$$
e_{D, 0}^{\tau \partial_{t}^{2}} \Phi_{0}=\Phi_{0} \operatorname{erf}\left(\frac{1}{2} \frac{t}{\sqrt{\tau}}\right)
$$

где

$$
\operatorname{erf}(t)=\frac{2}{\sqrt{\pi}} \int_{0}^{t} e^{-x^{2}} d x
$$

- функция ошибок.

Действуя теперь оператором $e_{D, 0}^{\tau \partial_{t}^{2}}$ на $\Phi_{0}+B_{0} \operatorname{sh}(r t)$, получим

$$
e_{D, 0}^{\tau \partial_{t}^{2}}\left(\Phi_{0}+B_{0} \operatorname{sh}(r t)\right)=\Phi_{0} \operatorname{erf}\left(\frac{1}{2} \frac{t}{\sqrt{\tau}}\right)+B_{0} e^{\tau r^{2}} \operatorname{sh}(r t) .
$$

Окончательно это соотношение может быть переписано в виде

$$
e_{D, 0}^{\tau \partial_{t}^{2}} \Phi=-J(\tau, t)+m^{2} \Phi,
$$

где

$$
J(\tau, t)=m^{2} \Phi_{0}-\Phi_{0} \operatorname{erf}\left(\frac{1}{2} \frac{t}{\sqrt{\tau}}\right), \quad \Phi(t)=\Phi_{0}+B_{0} \operatorname{sh}(r t)
$$

и

$$
r^{2}=\frac{\ln m^{2}}{\tau} .
$$

2.3. О нелинейных нелокальных уравнениях на полупрямой. Рассмотрим нелинейное нелокальное уравнение на полупрямой следующего вида:

$$
e_{D, J}^{\tau \partial_{t}^{2}} \Phi=V^{\prime}(\Phi(t)), \quad t>0
$$

где действие оператора $e_{D, J}^{\tau \partial_{t}^{2}}$ задаётся выражением (6) и потенциал взаимодействия $V(\Phi)$ имеет, например, вид $V(\Phi)=g \Phi^{4}$. Перейдя к пределу при $t \rightarrow 0$ в (11), получим важное соотношение

$$
\mu(\tau)=V^{\prime}(\Phi(0)) .
$$

Соотношение (12) показывает, что значение поля в начальный момент времени $\Phi(0)$ связано со значением функции $\mu$.

Было бы интересно исследовать разрешимость и свойства решений уравнения (11).

3. Нелокальные уравнения на полуоси во Фридмановской космологии. Рассмотрим действие вида

$$
S=\frac{1}{g_{4}} \int d^{4} x \sqrt{-g}\left\{m_{p}^{2} \frac{R}{2}+\frac{1}{\gamma}\left(\frac{1}{2} \Phi e^{-\frac{1}{4} \square} \Phi-V(\Phi)\right)\right\} .
$$

Здесь все координаты безразмерные, $m_{p}$ и $\gamma$ - безразмерные константы. 
Для пространственно плоской метрики Фридмана

$$
d s^{2}=-d t^{2}+a^{2}(t) d x^{2}
$$

динамика модели описывается системой из двух нелинейных нелокальных уравнений [1] для скалярного поля $\Phi(t)$ и параметра Хаббла $H(t)=\dot{a} / a$ :

$$
\begin{gathered}
e^{-\frac{1}{4} \mathcal{D}} \Phi=V^{\prime}(\Phi), \quad t>0 \\
3 H^{2}=\frac{1}{\gamma m_{p}^{2}}\left(\frac{1}{2} \Phi e^{-\frac{1}{4} \mathcal{D}} \Phi+V(\Phi)+\mathcal{E}_{1}+\mathcal{E}_{2}\right)
\end{gathered}
$$

где

$$
\mathcal{D}=-\partial_{t}^{2}-3 H(t) \partial_{t}
$$

и

$$
\begin{aligned}
\mathcal{E}_{1} & =\frac{1}{8} \int_{0}^{1} d s\left(e^{\frac{s-2}{8} \mathcal{D}} \Phi\right) \cdot\left(\mathcal{D} e^{-\frac{1}{8} s \mathcal{D}} \Phi\right) \\
\mathcal{E}_{2} & =\frac{1}{8} \int_{0}^{1} d s\left(\partial_{t} e^{\frac{s-2}{8} \mathcal{D}} \Phi\right) \cdot\left(\partial_{t} e^{-\frac{1}{8} s \mathcal{D}} \Phi\right) .
\end{aligned}
$$

Определим действие оператора $e_{D, \mu}^{-\tau \mathcal{D}}$ на полуоси $t>0$ (ср. с определением аналогичного оператора на всей оси в $[7,11,15,16])$ на функцию $\varphi(t)$ следующим образом:

$$
e_{D, J}^{-\tau \mathcal{D}} \varphi(t)=\Psi_{D, H}(t, \tau)
$$

где $\Psi_{D, H}(t, \tau)$ - решение задачи с граничным условием Дирихле:

$$
\left\{\begin{array}{l}
\frac{\partial}{\partial \tau} \Psi_{D, H}(t, \tau)=-\mathcal{D} \Psi_{D, H}(t, \tau), \quad t>0, \quad \tau>0 \\
\Psi_{D, H}(t, 0)=\varphi(t), \quad \Psi_{D, H}(0, \tau)=\mu(\tau) .
\end{array}\right.
$$

Рассмотрим случай $H(t)=H_{0}=$ const. Заметим, что решения первых уравнений в (13) и (5) связаны преобразованием

$$
\Psi_{D, H_{0}}(t, \tau)=e^{-\frac{3}{2} H_{0} t-\frac{9}{4} H_{0}^{2} \tau} \Psi_{D}(t, \tau) .
$$

Поэтому мы получаем решение уравнения (13) для $H(t)=H_{0}$ в виде

$$
\begin{array}{r}
\Psi_{D, H_{0}}(t, \tau)=e^{-\frac{3}{2} H_{0} t-\frac{9}{4} H_{0}^{2} \tau}\left[\frac{1}{\sqrt{4 \pi \tau}} \int_{0}^{\infty} e^{\frac{3}{2} H_{0} t^{\prime}} \varphi\left(t^{\prime}\right)\left[e^{-\frac{\left(t-t^{\prime}\right)^{2}}{4 \tau}}-e^{-\frac{\left(t+t^{\prime}\right)^{2}}{4 \tau}}\right] d t^{\prime}+\right. \\
\left.+\frac{t}{\sqrt{4 \pi}} \int_{0}^{\tau} \frac{e^{\frac{9}{4} H_{0}^{2} \tau^{\prime}} \mu\left(\tau^{\prime}\right)}{\left(\tau-\tau^{\prime}\right)^{3 / 2}} e^{-\frac{t^{2}}{4\left(\tau-\tau^{\prime}\right)}} d \tau^{\prime}\right] .
\end{array}
$$

Обозначив $\Psi_{D, H_{0}}(t, \tau)=e_{D, \mathcal{J}}^{-\tau \mathcal{D}} \varphi(t)$, имеем

$$
e_{D, \mathcal{J}}^{-\tau \mathcal{D}} \varphi(t)=e_{D, 0}^{-\tau \mathcal{D}} \varphi(t)+\mathcal{J}(\tau, t)
$$


где

$$
\mathcal{J}(\tau, t)=e^{-\frac{3}{2} H_{0} t-\frac{9}{4} H_{0}^{2} \tau} \frac{t}{\sqrt{4 \pi}} \int_{0}^{\tau} \frac{e^{\frac{9}{4} H_{0}^{2} \tau^{\prime}} \mu\left(\tau^{\prime}\right)}{\left(\tau-\tau^{\prime}\right)^{3 / 2}} e^{-\frac{t^{2}}{4\left(\tau-\tau^{\prime}\right)}} d \tau^{\prime}
$$

Действие оператора $e_{D, 0}^{-\tau \mathcal{D}}$ на функцию $e^{-\frac{3}{2} H_{0} t} \operatorname{sh}(r t)$ имеет вид

$$
e_{D, 0}^{-\tau \mathcal{D}} e^{-\frac{3}{2} H_{0} t} \operatorname{sh}(r t)=e^{\tau\left(r^{2}-\frac{9}{4} H_{0}^{2}\right)} e^{-\frac{3}{2} H_{0} t} \operatorname{sh}(r t)
$$

Аналогично можно определить действие оператора с граничными условиями Неймана $e_{N, \mu}^{-\tau \mathcal{D}}$ на функцию $\varphi(t)$ :

$$
e_{N, \nu}^{-\tau \mathcal{D}} \varphi(t)=\Psi_{N, H}(t, \tau)
$$

Здесь $\Psi_{N, H}(t, \tau)$ - решение задачи

$$
\left\{\begin{array}{l}
\frac{\partial}{\partial \tau} \Psi_{N, H}(t, \tau)=-\mathcal{D} \Psi_{N, H}(t, \tau), \quad t>0, \quad \tau>0 \\
\Psi_{N, H}(t, 0)=\varphi(t),\left.\quad \frac{\partial}{\partial t} \Psi_{N, H}(t, \tau)\right|_{t=0}=\nu(\tau)
\end{array}\right.
$$

При $H(t)=H_{0}$ решение задачи (15) имеет вид

$$
\begin{aligned}
& \Psi_{N, H_{0}}(t, \tau)=e^{-3 H_{0} t-\frac{9}{4} H_{0}^{2} \tau}\left[\frac{1}{\sqrt{4 \pi \tau}} \int_{0}^{\infty} e^{3 H_{0} t^{\prime}} \varphi\left(t^{\prime}\right)\left[e^{-\frac{\left(t-t^{\prime}\right)^{2}}{4 \tau}}+e^{-\frac{\left(t+t^{\prime}\right)^{2}}{4 \tau}}\right] d t^{\prime}+\right. \\
& \left.+\frac{1}{\sqrt{\pi}} \int_{0}^{\tau} \frac{e^{\frac{9}{4} H_{0}^{2} \tau^{\prime}} \nu\left(\tau^{\prime}\right)}{\left(\tau-\tau^{\prime}\right)^{1 / 2}} e^{-\frac{t^{2}}{4\left(\tau-\tau^{\prime}\right)}} d \tau^{\prime}\right]
\end{aligned}
$$

Действие оператора $e_{N, 0}^{-\tau \mathcal{D}}$ на функцию $e^{-\frac{3}{2} H_{0} t} \operatorname{ch}(r t)$ принимает вид

$$
e_{N, 0}^{-\tau \mathcal{D}} e^{-\frac{3}{2} H_{0} t} \operatorname{ch}(r t)=e^{\tau\left(r^{2}-\frac{9}{4} H_{0}^{2}\right)} e^{-\frac{3}{2} H_{0} t} \operatorname{ch}(r t)
$$

3.1. Решение линеаризованных уравнений при $\mathrm{H}_{0} \neq 0$. Рассмотрим линеаризованные уравнения с граничными условиями Дирихле с $J(t, \tau)=0$ :

$$
e_{D, 0}^{\tau \mathcal{D}} \Phi(t)=m^{2} \Phi(t), \quad t>0
$$

где $\tau>0$ - фиксированный параметр. Это уравнение имеет вид интегрального уравнения

$$
\frac{e^{-\frac{3}{2} H_{0} t-\frac{9}{4} H_{0}^{2} \tau}}{2 \sqrt{\tau \pi}} \int_{0}^{\infty} d t^{\prime} e^{\frac{3}{2} H_{0} t}\left[e^{-\frac{\left(t-t^{\prime}\right)^{2}}{4 \tau}}-e^{-\frac{\left(t+t^{\prime}\right)^{2}}{4 \tau}}\right] \Phi\left(t^{\prime}\right)=m^{2} \Phi(t), \quad t>0 .
$$

Используя (14), получаем, что

$$
\Phi(t)=B_{1} e^{-\frac{3}{2} H_{0} t} \operatorname{sh}(r t)
$$


является решением уравнения (16), если

$$
\left(r^{2}-\frac{9}{4} H_{0}^{2}\right)=\frac{\ln m^{2}}{\tau} .
$$

Энергия и давление для этого решения имеют вид

$$
\begin{gathered}
E_{D, H_{0}}\left(B_{1}\right)=-\left(r^{2}-\frac{9}{4} H_{0}^{2}\right) e^{\left(r^{2}-\frac{9}{4} H_{0}^{2}\right)} B_{1}^{2}, \\
P_{D, H_{0}}\left(B_{1}\right)=-\left(r^{2}-\frac{9}{4} H_{0}^{2}\right) B_{1}^{2} e^{\left(r^{2}-\frac{9}{4} H_{0}^{2}\right)} \operatorname{ch}\left(2 t \sqrt{r^{2}-\frac{9}{4} H_{0}^{2}}\right) .
\end{gathered}
$$

Получаем, что в режиме большого трения $r^{2} \ll H_{0}^{2}$ уравнение (17) имеет решение только для случая $m^{2}<1$. В этом случае энергия будет положительной и значение $H_{0}$ можно определить из уравнения

$$
3 H_{0}^{2}=\frac{1}{\gamma m_{p}^{2}} \frac{9}{4} H_{0}^{2} e^{\left(r^{2}-\frac{9}{4} H_{0}^{2}\right)} B_{1}^{2} .
$$

4. Инфляционная космология. В данной работе показано, что в определении нелокального оператора на полуоси имеется произвольная функция $($ источник $J$ ). Рассмотрим возможное использование этой функции в сценарии инфляционной космологии [42].

Космологические наблюдения показывают, что Вселенная пространственно почти плоская и возмущения плотности масштабно инвариантны, гауссовы и адиабатичны. Простейшее объяснение этих наблюдений даётся инфляционным сценарием с медленно скатывающимся в пологом потенциале скалярным полем.

В сценарии хаотической инфляции с исчезающей космологической постоянной достаточно реалистические результаты могут быть получены уже для простейшего потенциал вида $U(\phi)=m^{2} \phi^{2} / 2$. В этом сценарии предполагается, что ранняя Вселенная первоначально состояла из многих областей с хаотически распределенным скалярным полем. Затем быстрое расширение (инфляция) генерировало из областей, в которых скалярное поле было относительно большим, множество очень больших однородных областей. Наша наблюдаемая Вселенная является одной из этих областей.

Предполагается, что естественное начальное условие для скалярного поля $\phi_{0}$ в момент, когда классическое описание Вселенной уже становится применимым, есть $U\left(\phi_{0}\right) \lesssim M_{P}^{4}$, где $U(\phi)$ - потенциал и $M_{P}$ - планковская масса.

Желательно иметь инфляционный сценарий с малым значением $\phi_{0}$, так как имеются сомнения, что на планковских масштабах применимо классическое описание.

Рассмотрим действие (2). Уравнения Фридмана для однородного поля $\phi=$ $=\phi(t)$ и источника $J=J(t)$ имеют вид

$$
\ddot{\phi}+3 H \dot{\phi}+U^{\prime}(\phi)-J=0, \quad H^{2}=\frac{1}{3 M_{P}^{2}}\left(\frac{\dot{\phi}^{2}}{2}+U(\phi)-J \phi\right) .
$$

Параметры медленного скатывания суть

$$
\epsilon=\frac{M_{P}^{2}}{2}\left(\frac{U^{\prime}(\phi)-J}{U(\phi)-J \phi}\right)^{2}, \quad \eta=M_{P}^{2} \frac{U^{\prime \prime}(\phi)}{U(\phi)-J \phi} .
$$


Если потенциал $U(\phi)=m^{2} \phi^{2} / 2$, то

$$
\epsilon=\frac{M_{P}^{2}}{2 \phi^{2}}\left(\frac{m^{2} \phi-J}{\frac{m^{2}}{2} \phi-J}\right)^{2}=\frac{M_{P}^{2}}{2 \phi^{2}} \frac{\delta^{2}}{\left(\frac{m^{2}}{2} \phi-\delta\right)^{2}},
$$

где $\delta=m^{2} \phi-J$.

Поэтому в области пространства-времени, где функция источника $J$ такова, что $\delta \ll m^{2} \phi / 2$, мы получаем малый параметр $\epsilon$ даже для не очень больших значений поля $\phi . \mathrm{K}$ сожалению, параметр $\eta$ не удаётся сделать очень малым для малых значений $\phi$ при помощи выбора функции источника $J$, для этого, по-видимому, требуются новые идеи.

Работа выполнена при частичной поддержке РФФИ (проекты №o 11-01-00894-а (И.А.), 11-01-00828-а (И.В.)) и гранта президента РФ для поддержки ведущих научных школ (проекты НШ-8265.2010.1 (И.А.), НШ-7675.2010.1 (И.В.)).

\section{БИБЛИОГРАФИЧЕСКИЙ СПИСОК}

1. Aref'eva I. Ya. Nonlocal string tachyon as a model for cosmological dark energy // AIP Conf. Proc., 2006. Vol. 826. Pp. 301-311, arXiv: astro-ph/0410443.

2. Aref'eva I. Ya., Koshelev A.S., Vernov S. Yu. Crossing of the $w=-1$ barrier by D3-brane dark energy model// Phys. Rev. D, 2005. Vol. 72, no. 6, 064017, arXiv: astro-ph/0507067.

3. Calcagni $G$. Cosmological tachyon from cubic string field theory // JHEP, 2006. Vol. 2006, no. 05, 012. 25 pp., arXiv: hep-th/0512259

4. Barnaby N., Biswas T., Cline J. M. p-Adic inflation // JHEP, 2007. Vol. 2007, no. 04, 056. 35 pp., arXiv: hep-th/0612230

5. Aref'eva I. Ya., Joukovskaya L. V., Vernov S. Yu. Bouncing and accelerating solutions in nonlocal stringy models // JHEP, 2007. Vol. 2007, no. 07, 087. 36 pp., arXiv: hep-th/0701184

6. Calcagni G., Montobbio M., Nardelli G. Route to nonlocal cosmology // Phys. Rev. D, 2007. Vol. 76, no. 12, 126001. 20 pp., arXiv: 0705.3043 [hep-th]

7. Joukovskaya L. Dynamics in nonlocal cosmological models derived from string field theory // Phys. Rev. D, 2007. Vol. 76, no. 10, 105007. 12 pp., arXiv: 0707.1545 [hep-th]

8. Aref'eva I. Ya., Joukovskaya L.V., Vernov S. Yu. Dynamics in nonlocal linear models in the Friedmann-Robertson-Walker metric // J. Phys. A: Math. Theor., 2008. Vol. 41, no. 30, 304003, arXiv: 0711.1364 [hep-th].

9. Barnaby N., Cline J. M. Predictions for nongaussianity from nonlocal inflation // JCAP, 2008. Vol. 2008, no. 06, 030, arXiv: 0802.3218 [hep-th].

10. Aref'eva I. Ya., Koshelev A.S. Cosmological signature of tachyon condensation // JHEP. Vol. 2008, no. 09, 068. 20 pp., arXiv: 0804.3570 [hep-th]

11. Mulryne D. J., Nunes N.J. Diffusing nonlocal infation: Solving the feld equations as an initial value problem // Phys. Rev. D, 2008. Vol. 78, no. 06, 063519. 16 pp., arXiv: 0805.0449 [hep-th]

12. Joukovskaya L.V. Dynamics with infinitely many time derivatives in FriedmannRobertson0-Walker background and rolling tachyon // JHEP, 2009. Vol. 2009, no. $02,045$. 18 pp., arXiv: 0807.2065 [hep-th]

13. Barnaby $N$., Kamran N. Dynamics with infinitely many derivatives: Variable coefficient equations // JHEP, 2008. Vol. 2008, no. 12, 022. 27 pp., arXiv: 0809.4513 [hep-th]

14. Mulryne D.J., Nunes N.J. Non-linear non-local cosmology// AIP Conf. Proc., 2009. Vol. 1115. Pp. 329-334, arXiv: 0810.5471 [astro-ph].

15. Barnaby N. Nonlocal inflation // Can. J. Phys.. Vol. 87, no. 3. Pp. 189-194, arXiv: 0811.0814 [hep-th].

16. Calcagni G., Nardelli G. Nonlocal gravity and the diffusion equation // Phys. Rev. D, 2010. Vol. 82, no. 12, 123518. 17 pp., arXiv: 1004.5144 [hep-th]. 
17. Koshelev A.S., Vernov S. Yu. Analysis of scalar perturbations in cosmological models with a non-local scalar field // Class. Quantum Grav., 2011. Vol. 28, no. 8, 08501, arXiv: 1009.0746 [hep-th].

18. Galli F., Koshelev A.S. Perturbative stability of SFT-based cosmological models, arXiv: 1011.5672 [hep-th].

19. Aref'eva I. Ya. Puzzles with tachyon in SSFT and cosmological applications, arXiv: 1101.5338 [hep-th].

20. Aref'eva I. Ya., Volovich I. V. Cosmological daemon, arXiv: 1103.0273 [hep-th].

21. Volovich I. V. p-Adic string// Class. Quant. Grav., 1987. Vol. 4, no. 4, L83.

22. Brekke L., Freund P. G. O., Olson M., Witten E. Non-archimedean string dynamics // Nucl. Phys. B, 1988. Vol. 302, no. 3. Pp. 365-402.

23. Frampton P. H., Okada Ya. Effective scalar field theory of $p$-adic string // Phys. Rev. D, 1988. Vol. 37, no. 10. Pp. 3077-3079.

24. Владимиров В.С, Волович И. В., Зеленов Е.И. р-Адический анализ и математическая физика. М.: Физматлит, 1994. 352 с.; англ. пер.: Vladimirov V.S., Volovich I. V., Zelenov E. I. p-Adic analysis and mathematical physics. Vol.1/ Series on Soviet and East European Mathematics. River Edge, NJ: World Scientific Publishing Co., Inc., 1994. 319 pp.

25. Dragovich B., Khrennikov A. Yu., Kozyrev S. V., Volovich I. V. On p-adic mathematical physics // p-Adic Numbers, Ultrametric Analysis, and Applications, 2009. Vol. 1, no. 1. Pp. 117, arXiv: 0904.4205 [math-ph].

26. Владимиров В.С., Волович Я.И. О нелинейном уравнении динамики в теории $p$ адической струны // TMФ, 2004. Т. 138, №3. С. 355-368; англ. пер.: Vladimirov V.S., Volovich Ya.I.// Theor. Math. Phys., 2004. Vol.138, no.3. Pp. 297-309, arXiv: math$\mathrm{ph} / 0306018$.

27. Moeller N., Zwiebach B. Dynamics with infinitely many time derivatives and rolling tachyons // JHEP, 2002. Vol. 2002, no. 10, 034. 39 pp., arXiv: hep-th/0207107

28. Volovich Ya.I. Numerical study of nonlinear equations with infinite number of derivatives // J. Phys. A: Math. Gen., 2003. Vol. 36, no. 32, 8685, arXiv: math-ph/0301028.

29. Владимиров B. C. Об уравнении $p$-адической открытой струны для скалярного поля тахионов // Изв. РАН. Сер. матем., 2005. Т. 69, № 3. С. 55-80; англ. пер.: Vladimirov V.S. The equation of the $p$-adic open string for the scalar tachyon field // Izv. Math., 2005. Vol.69, no. 3. Pp. 487-512, arXiv: math-ph/0507018.

30. Владимиров В. С. К вопросу об асимптотике при $|t| \rightarrow \infty$ решений краевых задач для p-адических струн // TMФ, 2008. Т. 157, №3. С. 325-333; англ. пер.: Vladimirov V.S. The question of the asymptotic behavior as $|t| \rightarrow \infty$ of boundary value problem solutions for $p$-ADIC strings // Theoret. and Math. Phys., 2008. Vol. 157, no. 3. Pp. 1638-1645.

31. Gorka P., Prado H., Reyes E. G. Functional calculus via Laplace transform and equations with infinitely many derivatives // J. Math. Phys., 2010. Vol. 51, no. 10, 103512. 10 pp.

32. Davis H. T. The theory of linear operators from the standpoint of differential equations of infinite order / Monograph of the Waterman institute of Indiana university (contribution no. 72). Bloomington, Ind.: Principia Press, 1936. 628 pp.

33. Carleson L. On infinite differential equations with constant coefficients. I// Math. Scand., 1953. Vol. 1. Pp. 31-38.

34. Hörmander L. The analysis of linear partial differential operators. Vol. II: Differential operators with constant coefficients / Reprint of the 1983 Edition. Berlin, Heidelberg: Springer, 2005. 394 pp.

35. Арабаджян Л. Г., Енгибарян Н. Б. Уравнения в свертках и нелинейные функциональные уравнения / Итоги науки и техн. Сер. Мат. анал., Т. 22. М.: ВИНИТИ, 1984. С. 175244; англ. пер.: Arabadzhyan L. G., Engibaryan N. B. Convolution equations and nonlinear functional equations // J. Soviet Math., 1987. Vol.36, no. 6. Pp. 745-791.

36. Хачатрян A.X., Хачатрян $X$. A. Об одном нелинейном интегральном уравнении типа уравнения Гаммерштейна с некомпактным оператором // Матем. сб., 2010. Т. 201, № 4. C. 125-136; англ. пер.: Khachatryan A.Kh., Khachatryan Kh.A. A nonlinear integral 
equation of Hammerstein type with a noncompact operator // Sb. Math., 2010. Vol.201, no. 4. Pp. 595-606.

37. Hawking S., Ellis J. The large scale structure of space-time/ Cambridge Monographs on Mathematical Physics. Vol. I. Cambridge: Cambridge University Press, 1973. 391 pp.

38. Фок В. А. Собственное время в классической и квантовой механике // Изв. АН CCCP, серия физ., 1937. № 4-5. С. 551-568; нем. пер.: Fock V. Die eigenzeit in der klassichen und in der quantenmechanick // Phys. Z. Sowetunion, 1937. Vol. 12, no. 4. Pp. 404-425.

39. Feynman R.P. Mathematical formulation of the quantum theory of electromagnetic interaction // Phys. Rev., 1950. Vol. 80, no. 3. Pp. 440-457.

40. Pais A., Uhlenbeck G. E. On field theories with non-localized action// Phys. Rev., 1950. Vol. 79, no. 1. Pp. 145-165.

41. Владимиров В.С. Уравнения математической физики. М.: Наука, 1971. 512 с. [Vladimirov V.S. Equations of mathematical physics. Moscow: Nauka, 1971. 512 pp.]

42. Linde A. Inflation and Quantum cosmology. Boston, MA (USA): Academic Press, 1990 $211 \mathrm{pp}$

Поступила в редакцию 22/III/2011;

в окончательном варианте - 27/III/2011.

\section{MSC: 83F05}

\section{ON NONLOCAL COSMOLOGICAL EQUATIONS ON HALF-LINE}

\section{Ya. Aref'eva, I. V. Volovich}

Steklov Mathematical Institute, Russian Academy of Sciences, 8, Gubkina st., Moscow, 119991, Russia.

E-mails: arefeva@mi.ras.ru, volovich@mi.ras.ru

A system of nonlocal cosmological equations where the time variable runs over a halfline is considered. These equations are more suitable for description of the Universe than the previously discussed cosmological equations on the whole line since the Friedmann metric contains a singularity at the beginning of time. Definition of the exponential operator includes a new arbitrary function which is absent in the equations on the whole line. It is shown that this function could be choosen in such a way that one of the slow roll parameters in the chaotic inflation scenario can be made arbitrary small. Solutions of the linearized nonlocal equations on the half-line are constructed.

Key words: equations with an infinite number of derivatives, cosmological models, heat conduction equation.

Original article submitted $22 / \mathrm{III} / 2011$; revision submitted $27 / \mathrm{III} / 2011$.

Irina Ya. Aref'eva (Dr. Sci (Phys. \& Math.)), Professor, Leading Researcher, Dept. of Theoretical Physics. Igor' V. Volovich (Dr. Sci (Phys. \& Math.), Corresponding member of RAS), Head of Dept., Dept. of Mathematical Physics. 